\title{
Pseudoquiste adrenal. Aportación de un nuevo caso y revisión de la literatura
}

\author{
Sáenz Medina J, Esteban Peris A*, Linares Quevedo AI, Vallejo Herrador J, Castillón Vela I, \\ Páez Borda A.
}

Servicio de Urología. *Servicio de Radiología del Hospital de Fuenlabrada. Madrid.

Actas Urol Esp 2006; 30 (4): 420-423

\section{RESUMEN}

PSEUDOQUISTE ADRENAL. APORTACIÓN DE UN NUEVO CASO Y REVISIÓN DE LA LITERATURA Los pseudoquistes adrenales son las lesiones quísticas mas frecuentes de la glándula suprarrenal, si bien constituyen una rara entidad. Se detectan cada vez con mas frecuencia en estudios radiológicos como incidentalomas. Se presenta el caso de una paciente con pseudoquiste adrenal izquierdo no funcionante y asintomático. Se realiza revisión de la literatura y se estudian las diferentes actitudes diagnósticas y terapéuticas.

Palabras clave: Pseudoquiste suprarrenal. Quiste suprarrenal. Tumor suprarrenal

\section{ABSTRACT}

ADRENAL PSEUDOCYST. REPORT OF A NEW CASE AND REVIEW OF THE LITERATURE

Adrenal pseudocyst is the commonest type of benign lesions of adrenal gland althought is a very rare entity. Most of them are found as "incidentalomas" during imaging studies. A case of a patient with a left non-functioning and asymptomatic adrenal pseudocyst is reported . The diagnostic and therapeutic options are discussed and the literature is reviewed.

Keywords: Adrenal Pseudocyst. Adrenal cyst. Adrenal tumor.

$\mathrm{L}$ os quistes suprarrenales son una entidad infrecuente cuya incidencia varía entre el $0,064 \%$ y el $0,18 \%$ según las series de autopsias realizadas, habiéndose publicado menos de 500 casos en la literatura ${ }^{1}$. En nuestro país existen varias publicaciones de casos aislados siendo la serie más importante la de Deus Fondebilla ${ }^{3}$ que presenta cinco casos. La mayoría se diagnostican como incidentalomas, ocurriendo más frecuentemente en mujeres entre la cuarta y la quinta década de la vida.

Histológicamente se dividen en cuatro grupos: parasitarios, epiteliales (verdaderos quistes), endoteliales (quistes vasculares con revestimiento endotelial) y pseudoquistes ${ }^{2}$. Existen otros subtipos más infrecuentes como los linfangiomas, los quistes mesoteliales o los dermoides.
El pseudoquiste es el subtipo mas frecuente, carece de revestimiento epitelial, representa un residuo encapsulado de una hemorragia suprarrenal, pudiéndose encontrar calcificaciones en el $10-15 \%$ de los casos, no implicando este hecho malignidad.

La mayoria cursan asintomáticos y se descubren de forma incidental; cuando son sintomáticos suele ser derivado de la compresión de estructuras vecinas o por sangrado intraquístico. Los síntomas más habituales ${ }^{4}$ son la masa abdominal, el dolor abdominal o lumbar, o los síntomas gastrointestinales. La fiebre y el dolor son sintomas derivados de la infección o la hemorragia intraquística. Otros sintomas más infrecuentes son la hipertensión arterial $^{4}$ o la ruptura espontánea el mismo con hemorragia retroperitoneal consecutiva ${ }^{5}$. 
El diagnóstico se realiza con TAC, cuyos hallazgos ${ }^{7}$ suelen ser de una tumoración pequeña y bien definida con valor de atenuación similar al agua y homogéneo, apareciendo calcificaciones en su pared en el 15\% de los casos. Se deben realizar algunas determinaciones analiticas como potasio sérico o cortisol y metanefrinas en orina de 24 horas que faciliten el diagnóstico diferencial con otras entidades.

El tratamiento sigue siendo tema de controversia, aunque parece que en lesiones pequeñas asintomáticas se debe optar por la observación. Presentamos un caso de pseudoquiste adrenal asintomático diagnosticado en nuestro hospital y discutimos posteriormente las posibilidades de tratamiento.

\section{CASO CLÍNICO}

Una mujer de 48 años de edad fue remitida a nuestra consulta para valoración de dolor en fosa renal derecha. Una ecografía rutinaria evidenció una lesión quística en el polo superior del riñón izquierdo. La enferma no refería clínica sistémica ni miccional acompañante.

El TAC abdominal (Figs. 1 y 2) evidenció una lesión localizada en la glándula suprarrenal izquierda con calcificaciones periféricas y sin áreas de captación de contraste intravenoso.

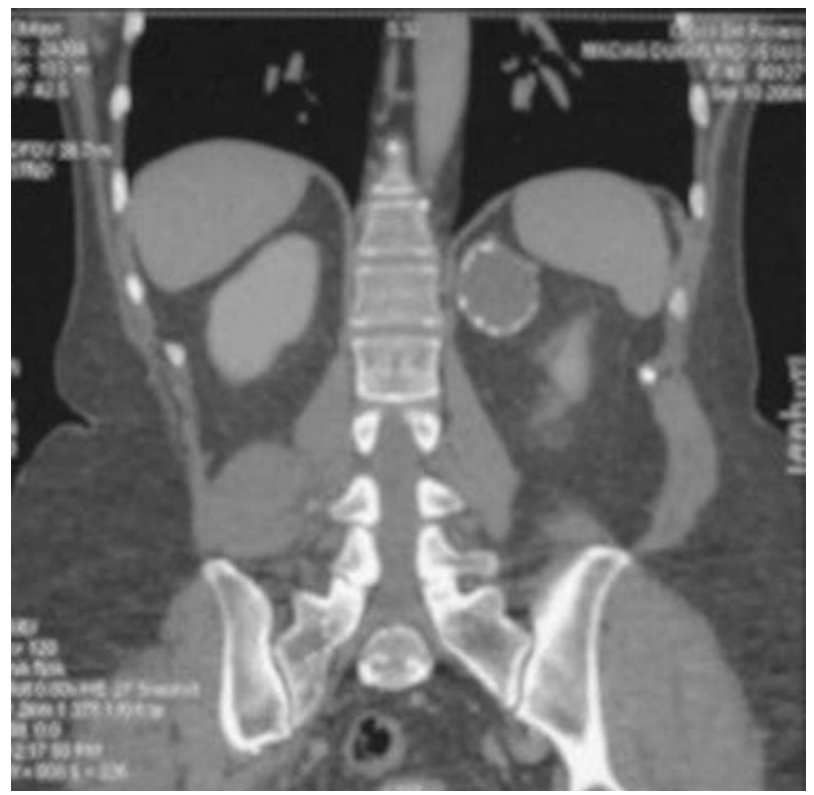

FIGURA 1: Reconstrucción coronal de TAC abdominal en la que se evidencia lesión en hipocondrio izquierdo con calcificaciones periféricas.

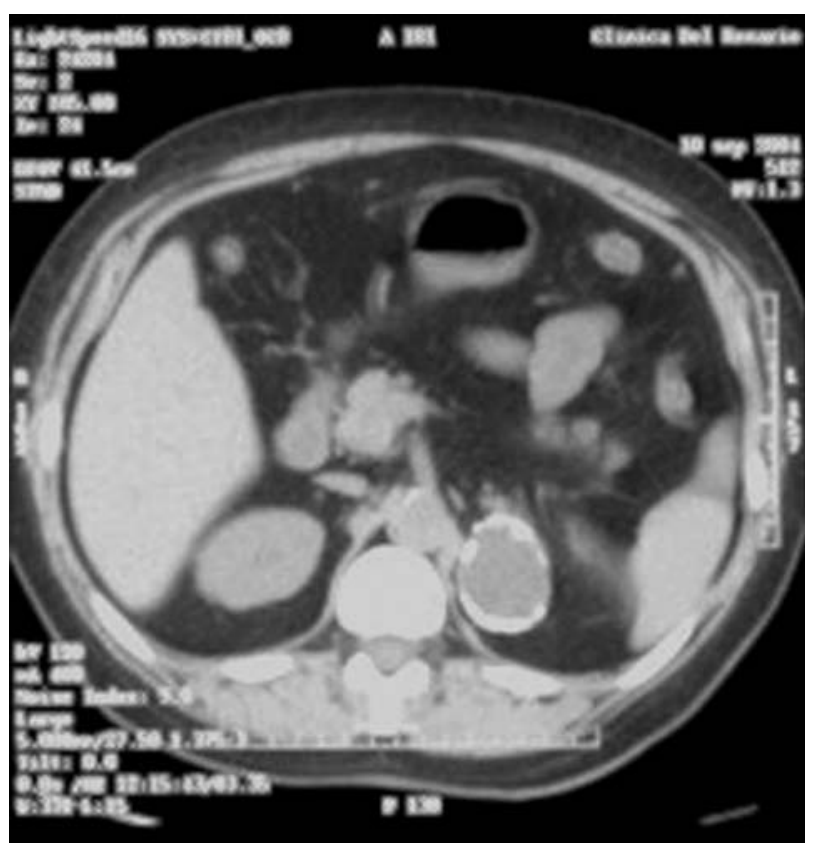

Figura 2: Corte axial con contraste intravenoso sin areas de captación a nivel de la lesión, demostrando su naturaleza quistica.

La RMN fue informada como compatible con una masa quística de $45 \mathrm{mms}$ con cápsula gruesa hipointensa en T2 (Fig. 3) no captante de Gadolinio (Figs. 4 y 5) y que no perdía señal en fuera de fase. El hemograma y la bioquímica $\mathrm{y}$ los iones (NA, $\mathrm{K}$ y $\mathrm{Cl}$ ) fueron normales.

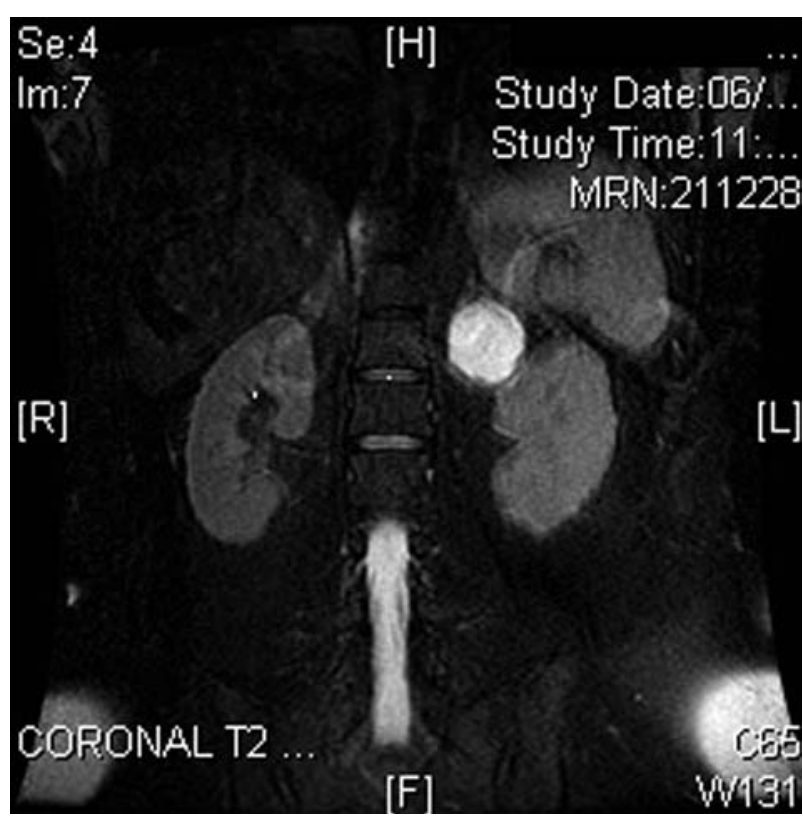

FIGURA 3: RMN secuencia T2. Corte coronal de lesion hiperintensa localizada en polo superior renal izquierdo. 


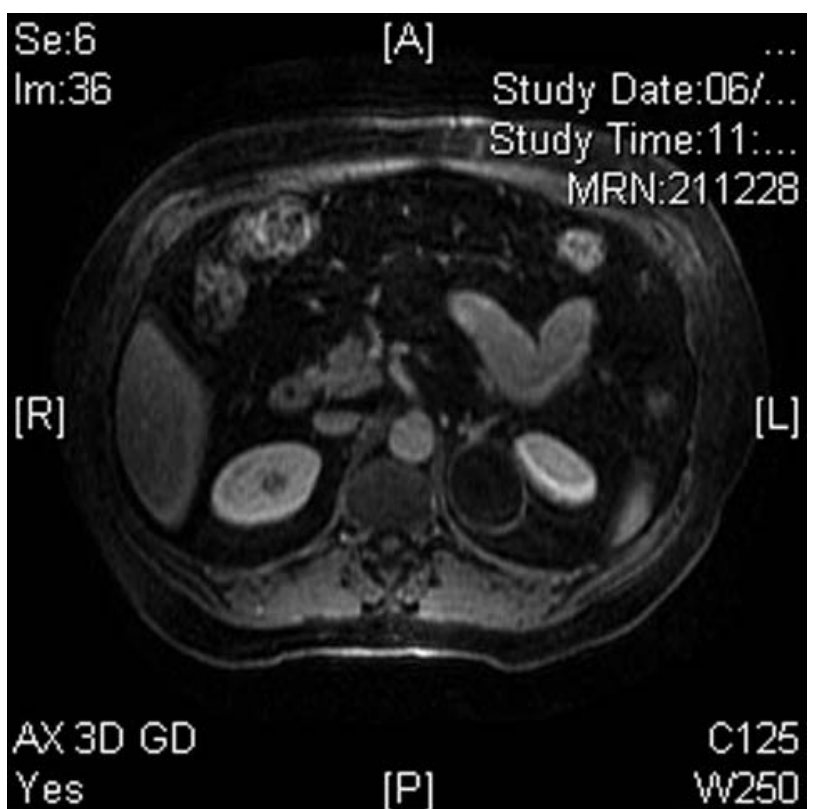

FIGURA 4: Corte axial de la lesión tras la administración de gadolinio.

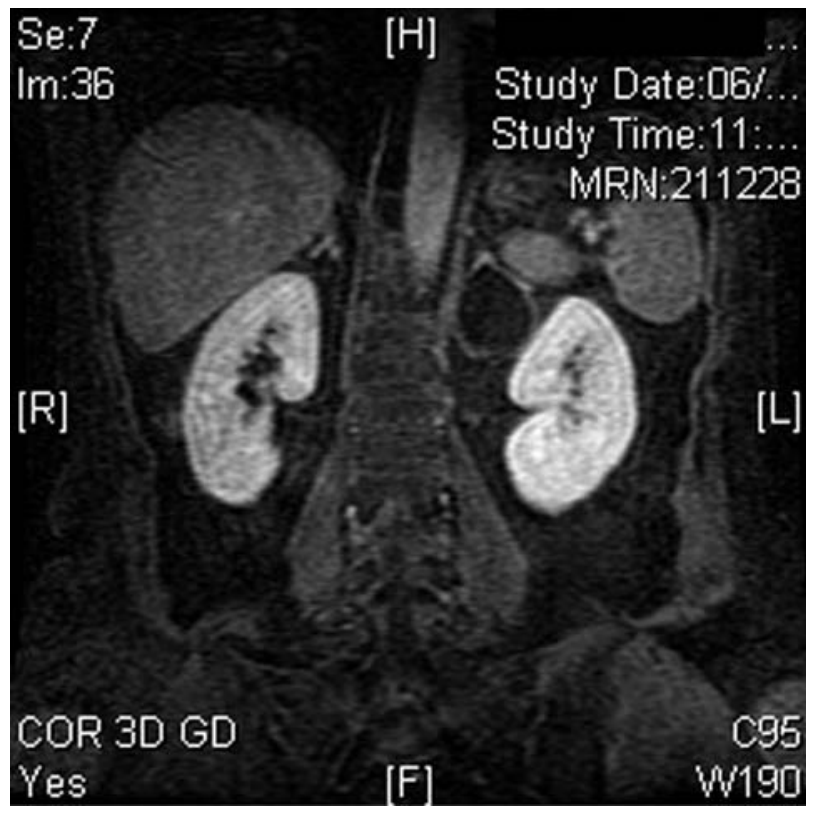

FIGURA 5: RMN corte coronal con gadolinio. Corte de lesión no captante con cápsula gruesa calcificada.

El sedimento y las metanefrinas y catecolaminas en orina se encontraron dentro de límites normales. La coagulación presentó una APTT ligeramente alargada $(38,1 \mathrm{~s})$, con actividad de factor VIII disminuida (43\%) y Ag factor Von Willebrand disminuido $(58,9)$, pendiente de estudio en hematología por dichas alteraciones de la coagulación.

\section{DISCUSIÓN}

La frecuencia con que se detectan los pseudoquistes adrenales depende de su índice de sospecha. En nuestro caso, como en la mayoría de los casos, el hallazgo fue incidental, en el contexto del estudio de un dolor lumbar contralateral de características mecánicas. El ionograma y las determinaciones de metanefrinas y catecolaminas en orina normales descartaron una masa suprarrenal funcionante. La alteración en la coagulación sugirió la hipótesis del pseudoquiste renal como reducto de una hemorragia suprarrenal antigua ${ }^{6}$.

Los hallazgos radiológicos en nuestro caso son superponibles a los descritos en la literatura, donde se describen como masas quísticas con contenido líquido y coeficiente de atenuación similar al agua, que en nuestro caso presentó calcificaciones en su pared, como ocurre hasta en el $15 \%$ de los $\operatorname{casos}^{7}$.

Existe controversia en cuanto al tratamiento mas adecuado de estas lesiones. Los hallazgos radiológicos pueden ser de gran ayuda en la decisión de la actitud terapéutica sin necesidad de biopsiar ni puncionar la masa para establecer la naturaleza histológica del proceso.

Rotzenbit $^{8}$ clasifica en cuatro tipos las masas adrenales quísticas no funcionantes basándose en criterios radiológicos: Se consideran quistes no complicados aquellos menores de 5-6 cm homogéneos, y con pared menor de $3 \mathrm{~mm}$; en esta variedad se recomienda la vigilancia periódica para detectar cambios en la naturaleza o en el tamaño. Como quistes complicados se clasifican aquellos con altos valores de atenuación o no homogéneos, con pared mayor de $5 \mathrm{~mm}$ de espesor o con gruesas calcificaciones centrales o periféricas: se recomienda su exéresis quirúrgica. Los quistes mayores de 5-6 cm con valores de atenuación mayores que el agua o con pared entre 3 y $5 \mathrm{~mm}$ se consideran indeterminados. En esta variedad estaría indicada una punción de la lesión previa a la decisión terapéutica.

Ansari $^{9}$ propone una clasificación clínica en función de los sintomas que producen: ante los asintomáticos propone vigilancia radiológica con TAC cada 6 meses, y exéresis sólo si reproducen aumentos de tamaño en el seguimiento; ante los sintomáticos y /o funcionantes sugiere exéresis de primera intención. 
La masa descrita en esta comunicación fue de $45 \mathrm{~mm}$ de diámetro, con una cápsula entre 3 y 4 $\mathrm{mm}$ en todos los cortes; fue diagnosticada de forma accidental y resultó afuncionante. En consecuencia, se decidió la observación con TAC y analítica cada 6 meses, no habiendo cambiado su aspecto en la segunda revisión. En el momento de redacción de estas líneas ( 8 meses tras el diagnóstico) el aspecto de la lesión no ha variado, motivo por el cual la paciente sigue en programa de observación.

\section{REFERENCIAS}

1. Bellantone R, Ferrante A, Raffaelli M, Boscherini M, Lombardi CP, Crucitti F. Adrenal cystic lesions: Report of 12 surgically treated cases and review of the literature. J Endocrinol Invest 1998;21(2):109-114.

2. Gaffey MJ, Mills SE, Fechner RE, Bertholf MF, Allen MS Jr. Vascular adrenal cysts: a clinicopathologic and immunohistochemical study of endothelial and hemorrhagic (pseudocystic) variants. Am J Surg Pathol 1989;13:740-747.

3. Deus Fombellida J, Gil Fabra J, Noguera Lobera A. Consideraciones sobre quistes y pseudoquistes suprarrenales. Arch Esp Urol 1986;39:301-312.
4. Karayiannakis AJ, Polychronidis A, Simopoulos C.: Giant adrenal pseudocyst presenting with gastric outlet obstruction and hypertension. Urology 2002 Jun;59(6):946.

5. Favorito LA, Lott FM, Cavalcante AG.: Traumatic rupture of adrenal pseudocyst leading to massive hemorrhage inretroperitoneum. Int Braz J Urol 2004 Jan-Feb;30(1):35-36.

6. Medeiros LJ, Lewandrowski KB, and Vickery AL Jr. Adrenal pseudocyst: a clinical and pathologic study of eight cases. Hum Pathol 1989;20:660-665.

7. Mc Loughlin RF, Bilbey JH. Tumors of the adrenal gland: findings on CT and MRI imaging. AJR 1994;18:432-438.

8. Rotzenblit A, Morehouse HT, Amis ES. Cystic adrenal lesions: CT features. Radiology 1996; 201:541-548.

9. Ansari MS, Singh I, Hemal AK. Cost-reductive retroperitoneal excision of large adrenal pseudocyst: A case report and review of the literature. Int Urol and Nephrol; Jun 2001;33(2):307-310.

Dr. J. Sáenz Medina.

E-mail: javiersaenzmedina@yahoo.es

(Trabajo recibido el 21 de junio de 2005) 